\title{
GDF5 modifies disease in OA rat model
}

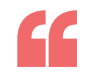

Rats treated with rhGDF5 showed visual improvement in their cartilage architecture
Growth and differentiation factor 5 (GDF5) could halt and reverse osteoarthritis (OA) progression, according to a new study published in Osteoarthritis and Cartilage. Recombinant human GDF5 (rhGDF5) had protective effects despite cartilage pathology being well-established before treatment in rats with surgically induced OA. "The potentially most significant finding of this work is that it clearly demonstrates the plausibility of a disease modifying osteoarthritic drug that can intercept and potentially reverse degenerative OA changes," comments William Parrish, corresponding author of the study.

"We have long suspected that GDF5 may hold significant potential

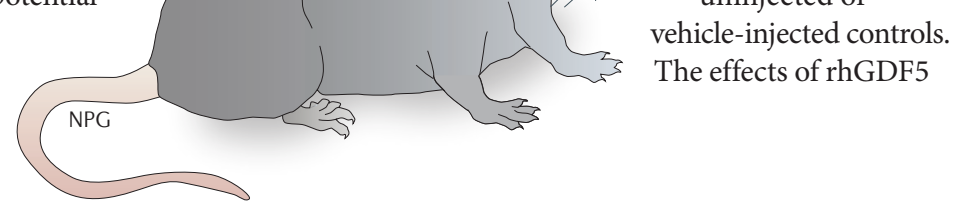

as a therapeutic agent for osteoarthritis," explains Parrish. "There is a considerable foundation of data that implicate a key role for GDF5 during the establishment of hyaline cartilage and in the maintenance of articular cartilage," he continues.

The investigators injected rhGDF5 into rats 21 days after medial meniscus transection (MTT) surgery, and measured histological changes within the joints 9 weeks post-surgery. Rats treated with rhGDF5 showed visual improvement in their cartilage architecture, increased medial collateral ligament thickness and decreased cartilage damage (as assessed by the width of cartilage lesions and cartilage matrix degeneration) compared with uninjected or vehicle-injected controls. The effects of rhGDF5 were dose-dependent: a single dose of $100 \mu \mathrm{g}$ rhGDF5 slowed disease progression, whereas two bi-weekly $100 \mu \mathrm{g}$ injections halted progression and three bi-weekly $100 \mu \mathrm{g}$ injections stimulated cartilage repair.

The MMT model is useful for observing structural changes in $\mathrm{OA}$, mirroring many disease aspects seen in humans. "We must remain mindful that success in this rat model of OA is not necessarily predictive of clinical success in such a multi-factorial and chronic disease as OA," points out Parrish. "For this reason, it is important to pursue multiple parallel paths towards clinical success," he concludes.

Jessica McHugh

ORIGINAL ARTICLE Parrish, W. R. et al. Intra-articular therapy with recombinant human GDF5 arrests disease progresion and stimulates cartilage repair in the rat medial meniscus transection (MMT) model of osteoarthritis,

Osteoarthritis Cartilage http://dx.doi. org/10.1016/j.joca.2016.11.002 (2016) 